\title{
Stream of consciousness ou points de confluence? Temporalité et conscience dans Mrs Dalloway : une approche psycholinguistique et cognitive
}

\section{Julie Neveux}

\section{(2) OpenEdition \\ Journals}

Édition électronique

URL : http://journals.openedition.org/esa/743

DOI : 10.4000/esa.743

ISSN : 2650-2623

Éditeur

Société de stylistique anglaise

Édition imprimée

Date de publication : 1 décembre 2016

Pagination : 83-104

ISBN : 978-2-36442-075-5

ISSN : 2116-1747

Référence électronique

Julie Neveux, «Stream of consciousness ou points de confluence? Temporalité et conscience dans Mrs Dalloway: une approche psycholinguistique et cognitive », Études de stylistique anglaise [En ligne], 10 | 2016, mis en ligne le 19 février 2019, consulté le 21 avril 2019. URL : http:// journals.openedition.org/esa/743; DOI : 10.4000/esa.743 


\section{Stream of consciousness ou points de confluence? Temporalité et conscience dans Mrs Dalloway : une approche psycholinguistique et cognitive}

Julie NEVEUX

Université Paris IV Sorbonne

CeLiSo, EA7332

\section{Introduction}

\section{Le temps lancinant}

Ce n'est que peu de temps avant d'envoyer son manuscrit, jusque-là le plus souvent désigné sous le titre The Hours, à son éditeur, en janvier 1925, que Woolf se décide à le renommer Mrs Dalloway. Le temps, qui passe, ne passe pas, que les coups de toutes les horloges de Londres, Big Ben en tête, s'obstinent à faire exister, est objet d'achoppement et/ou de fascination pour les personnages principaux du roman, et modèle la conscience qu'ils ont d'eux-mêmes : Clarissa ne cesse de revenir sur son âge, 51 ans dont trois mois restent encore entiers (D 40), la noix narrative imagine les antiquaires du futur "tamisant les ruines du temps " (sifting the ruins of time, D 17), Septimus est saisi d'un délire extatique lorsque sa femme prononce le mot "time" et se met à chanter une "ode immortelle au Temps ", avec une majuscule (an immortal ode to Time, D 76), dans l'esprit de Peter Walsh le temps stagne et "claque contre le mat " (Time flaps on the mast, D 54) et la cloche de St Margaret emprunte soudain les traits tant aimés de Clarissa. Les références au temps, chronologique ou vécu, extérieur ou intérieur, envahissent la diégèse : on compte dix occurrences du nom propre Big Ben, vingt-quatre du lemme clock, 
quarante du lemme hour, soixante-huit du lemme moment, quatre-vingtquinze du lemme time ${ }^{1}$.

Souvent la prise de conscience du temps s'accompagne d'un travail de mémoire. Ainsi l'emploi récurrent du verbe remember (vingt-huit occurrences) semble être une caractéristique spécifique de Mrs Dalloway, si on compare cet emploi à ceux relevés dans les cinq autres romans de Woolf écrits à la même époque ${ }^{2}$. Par quatre fois, tout au long du roman, la mention du temps qui s'écoule est suivie, en co-occurrence, de la proposition métaphorique The leaden circles dissolved in the air. Et cette métaphore des cercles de plomb qui se dissolvent, référant au son des cloches et à leur "irrévocabilité ", relie les inconscients des différents protagonistes : elle apparaît la première fois dans l'espace mental de Clarissa, la deuxième fois la focalisation porte sur Peter Walsh, la troisième sur Rezia, la femme de Septimus, et la dernière revient à Clarissa.

En tant que phénomène dont les manifestations affectent toutes les pensées, perceptions et sentiments des personnages, le temps est une donnée essentielle du roman. Une approche phénoménologique, cognitive et psycholinguistique peut tenter de rendre compte de l'interaction entre " expérience vive" du temps, pour reprendre les termes de Ricoeur (1984, 154, je souligne), et représentation de la conscience. Le son du temps est un quale dont on verra que paradoxalement il peut se partager. Woolf décrivait avec bonheur sa découverte stylistique dans son Journal, découverte consistant à dire le passé par fragments en creusant des "tunnels" qui finiraient par relier les "cavernes" situées "derrière" les personnages :

I should say a good deal about The Hours and my discovery: how I dig out beautiful caves behind my characters (...) the caves shall connect (...) It took me a year's groping to discover what I call my tunnelling process, by which I tell the past by instalments.

(W 1959, 60-61)

1 Tous les chiffres donnés ici sont obtenus grâce au logiciel TXM, disponible à http://textometrie.ens-lyon.fr/spip.php?article60 ; je remercie tous les membres de l'équipe de Lyon qui continuent à y travailler et à dispenser des formations précieuses.

2 Le corpus de comparaison est constitué des textes suivants : The Voyage Out (1915), Night and Day (1919), Jacob's Room (1922), To the Lighthouse (1927) et Orlando (1928). Le score de spécificité donné par TXM est très élevé : 6,4, i.e sur 310 occurrences dans l'ensemble du corpus, dont 65 dans Mrs Dalloway. 
La perception du temps est donc un processus central du courant de conscience dans Mrs Dalloway. Mais, de même que le temps vécu n'est pas linéaire, la technique même élaborée par Woolf ne nous livre pas d'accès immédiat à un flux de conscience qui serait brut et continu. Comme le montre De Mattia $(2001,228)$, cette définition classique de la technique du stream of consciousness ne convient pas ici, car la voix narrative travaille en permanence la représentation de la conscience des personnages.

Le récit fait pourtant place, lorsque le style figure ${ }^{3}$ l'expérience du temps, à des surgissements de conscience, dont je montrerai qu'ils constituent des points de confluence entre flux temporels; ces points de confluence sont réalisés par certaines métaphores ${ }^{4}$ récurrentes, qui assurent ainsi une cohérence sensorielle à l'inconscient personnel ou collectif (distinction qui n'est pas pertinente pour Woolf); le soi n'existe dans le temps que lorsque nul sujet, grammatical ou de conscience, ne le revendique.

\section{Au contact de l'eau : le flux interrompu}

Pourquoi la même métaphore leaden circles dissolved in the air émane-t-elle des consciences de personnages différents ? Toute-puissance narrative censurant l'individualité des personnages ${ }^{5}$ ? Characters are to be merely views: personality must be avoided at all costs, déclare en effet Woolf dans son Journal (W 1959, 60). Je ne mènerai pas ici d'analyse narratologique mais suivrai la piste psycholinguistique et cognitive, et m'appuierai, pour interpréter Mrs Dalloway, sur quelques autres écrits de Woolf fictifs et non fictifs, ainsi que sur certaines notions cognitives récentes.

3 Ricoeur présente Mrs Dalloway comme "une fable sur le temps" (1984, 152) dont l'écriture revient à "refigurer dans la lecture le temps lui-même" (1984, 157). La notion de figure, essentielle chez l'auteur de La métaphore vive (1975), est intrinsèquement liée à l'effet métaphorique, par différence avec la non-figuration de la comparaison (cf Neveux 2013, 97-116). Le pouvoir "figuratif" de la métaphore est ici exploité avec succès par Woolf, qui ne s'intéresse au temps qu'en tant que phénomène perçu par les personnages.

4 Le postulat de cette approche, que je ne présente pas ici, est la motivation expérientielle de la métaphore, et conséquemment, la faculté de cette figure de style à exprimer ce que j'appelle un "lyrisme indirect " motivé par une forte implication cognitive du sujet parlant (approche inspirée de la cognition dite incarnée, 'embodied' cognition et de la psychomécanique du langage, cf à ce propos Neveux 2013, 3014).

5 L'hypothèse est développée par De Mattia (2001, 261), qui cite Pascaud $(1988,116)$ dans "La grammaire du discours indirect libre et le sujet", Travaux du Cierec, LXX1, Domaine anglais. Saint Etienne, Université de Saint Etienne, p. 105-123. 
Le temps, s'il ne se manifeste en sons d'horloge ou rides au coin des yeux, n'existe pas. Il n'est qu'en se rendant perceptible. C'est pourquoi la saisie linguistique (mais aussi conceptuelle et existentielle) du temps ne peut être que métaphorique. Les linguistes cognitivistes Lakoff (1980, 1993, 218) et Johnson ont montré que le temps était souvent conçu à partir de l'expérience spatiale. On avance dans le temps comme on avance sur un chemin, c'est le présent, le chemin parcouru se situe derrière nous, c'est le passé, et le chemin restant à parcourir devant, c'est le futur. Il s'agit donc, dans leur terminologie d'origine, d'une métaphore structurelle et "orientationnelle", prêtant des caractéristiques de repérage spatial à une entité abstraite. Mais la façon dont Woolf métaphorise le temps dans le roman n'est pas tant spatiale que sonore, linéaire que discontinue : c'est le son, dans Mrs Dalloway, qui fait ressentir le passage du temps ; le temps est son, ou il n'est pas. Or, le plus souvent, lorsqu'il est son, il se propage sous forme d'onde, de vague, inonde les pièces et élargit les consciences.

Il y a de la dissolution dans le roman, de la séparation de matière qui devient liquide, de nombreuses inondations et plongeons, la plupart ${ }^{6}$ métaphoriques. What a plunge! s'exclame intérieurement Clarissa qui sort de chez elle au petit matin londonien, à l'incipit du récit. Les métaphores disant le contact souvent brutal avec l'eau (plunge, flood, sink, dive, etc) surviennent lorsqu'émerge un état de conscience spécifiquement lié à une forte exposition cognitive des personnages à leur environnement.

William James (1890, 1923), dont l'oeuvre inspira Woolf, insistait sur la pertinence de sa métaphore fluviale du stream pour définir le mode de manifestation, fluide, de la conscience :

Consciousness, then, does not appear to itself chopped up in bits. Such words as 'chain' or 'train' do not describe it fitly as it presents itself in the first instant. It is nothing jointed: it flows. A 'river' or a 'stream' are the metaphors by which it is most naturally described. In talking of it hereafter, let us call it the stream of thought, of consciousness, or of subjective life.

Or la linéarité qu'implique la métaphore du flux ne s'applique pas à l'écriture de Woolf telle qu'elle représente le fonctionnement de la conscience dans Mrs Dalloway : comme une présence à soi interrompue, intermittente, inexistante ou existant dans l'altérité. La métaphore du

6 Sur sept occurrences du lexème plunge dans le roman, six sont métaphoriques. 
stream a donc le désavantage d'occulter une autre dimension de la conscience, que les expériences stylistiques woolfiennes nous permettent de percevoir, et que le neurologue Damasio décrit comme un état de conscience étendu (extended consciousness). Cet état semble être stimulé dans le roman par une manifestation brutale du temps, dont le son évoque une eau qui se répand brutalement :

The sound of Big Ben flooded Clarissa 's drawing-room. (D 128)

(...) And the sound of the bell flooded the room with its melancholy wave. (D 129)

Dans le passage suivant, le temps est matérialisé comme une entité compacte dont on peut mesurer - "toucher " - les constituants/mois, et dont on peut in extremis "saisir " (deuxième métaphore, grasp, indiquant l'appréhension corporelle du temps devenu liquide) une goutte :

She was not old yet. She had just broken into her fifty-second year. Months and months of it were still untouched. June, July, August! Each still remained almost whole, and, as if to catch the falling drop, Clarissa (crossing to the dressing-table) plunged into the very heart of the moment. (D 40)

La métaphore nominale de la goutte est encore utilisée lorsque c'est Peter Walsh qui éprouve la beauté du moment :

Life itself, every moment of it, every drop of it, here, this instant, now, in the sun, in Regent 's Park, was enough. Too much indeed. (D 87)

Soit le temps n'existe pas, soit il s'expérimente en un moment isolé où il fait du goutte à goutte (the falling drop), est suspendu (cette pause, ce suspense que ressent Clarissa juste avant les coups de Big Ben, D 4), où l'horizon conscient s'élargit pour accueillir différents flux temporels. Le présent n'a de fait pas plus de réalité que le passé ou le futur, comme Woolf l'explique dans son essai The Moment: Summer's Night (CE 1966, 293) :

Yet what composed the present moment? If you are young, the future lies upon the present, like a piece of glass, making it tremble and quiver. If you are old, the past lies upon the present, like a thick glass, making it waver, distorting it. All the same, everybody believes that the present is something, seeks out the different elements in this situation in order to compose the truth of it, the whole of it. 
L'existence même du présent est problématique, seule une perception accrue d'un certain passage du temps signale l'appartenance de l'être à un tout en mutation/devenir esthétique. Il semble alors plus adéquat de parler de la conscience non comme un flux, mais comme la surgie intermittente de points de confluence temporels donnant une assise sensorielle au soi.

\section{'Moments of being' : la conscience élargie}

\section{Le choc existentiel : "ravissement " et supplément de sens}

Dans son essai autobiographique $A$ Sketch of the Past, Woolf distingue ce qu'elle appelle les "moments d'être/étant" (moments of being ${ }^{7}$ ), moments vécus avec intensité et en pleine conscience, des moments plus nombreux et banals de non-être (étant), moments of nonbeing :

Every day includes much more non-being than being (...) These separate moments of being were however embedded in many more moments of nonbeing. (...) A great deal of every day is not lived consciously. (MB 1976, 70)

Ces moments d'être que la romancière retranscrit dans ses fictions peuvent être suscités par des stimuli externes - coups de Big Ben, mouvement des feuilles dans les arbres, ou bien, dans la nouvelle intitulée 'Moments of Being', publiée en 1928, où apparaît l'expression pour la première fois, simple phrase aux accents surréalistes décrochant du sens utilitaire des mots 'Slater's Pins have No Points' $(1989,215)$; ou par des stimuli internes comme le souvenir multi-sensoriel des vagues qui se brisent sur le sable. C'est en effet un souvenir fondamental, institué par Woolf fondement mémoriel de toute sa vie dans ce même essai, qu'un de ces premiers moments d'être constitué par le fracas (vue et son étant mêlés, insiste l'auteure) des vagues, sentiment de pure "extase ", dont la réalité sensorielle, chaque fois qu'elle l'évoque, est plus grande que celle du moment présent, et où le sujet disparaît pour n'être plus que sensation et sentiment, ouverture aux éléments :

7 Traduit par Colette-Marie Huet chez Stock par «Instants de vie » en 2006. 
Those moments-in the nursery, on the road to the beach-can still be more real than the present moment. (...) I am hardly aware of myself, but only the sensation. I am only the container ${ }^{8}$ of the feeling of ecstasy, of the feeling of rapture. (MB 1976, 66-67)

Les mêmes termes, ecstasy et rapture, tous deux désignant un rapt, arrachement, une sortie de soi violente qui est aussi dite " révélation", caractérisent tour à tour ces moments d'intensité expérientielle dans Mrs Dalloway. Dans l'extrait ci-dessous, Clarissa dit l'intensité du moment où elle s'abandonne à ressentir ce que ressentent les hommes pour les femmes :

Only for a moment; but it was enough. It was a sudden revelation, a tinge like a blush which one tried to check and then, as it spread, one yielded to its expansion, and rushed to the farthest verge and there quivered and felt the world come closer, swollen with some astonishing significance, some pressure of rapture, which split its thin skin and gushed and poured with an extraordinary alleviation over the cracks and sores! (D 34-35)

Le monde acquiert, lors de ces moments, du sens (astonishing significance); ce sens a une vertu thérapeutique, tout comme les images de l'eau, ici gushed et poured, véhiculent souvent dans l'écriture de Woolf un sentiment d'apaisement, où la réalité s'éprouve et se déverse enfin : in floods reality (1976, 122, je souligne). Le sujet de l'expérience devient partie d'un tout signifiant si et seulement s'il est capable de s'ouvrir à ce choc expérientiel, voire d'en faire œuvre d'art: the shock-receiving capacity is what makes me a writer (MB 1976, 72 ) ; ce choc est la preuve qu'une réalité existe derrière les apparences, a token of some real thing behind appearances (MB 1976, 72).

\section{Métaphore primaire et modélisation : vers un schéma esthétique et cognitif ('pattern' et 'scene making')}

La valeur de ces moments tient au sens qu'ils donnent à notre vie ; ils sont un supplément de sens, que Woolf décrit comme un motif, un

\footnotetext{
8 Woolf utilise dans ce passage ce que Lakoff et Johson (1980) ont identifié comme la métaphore du "CONTENANT", où l'être humain se perçoit comme un réceptacle pouvant contenir des éléments divers. Fait paradoxal en apparence, la métaphore exprime ici précisément un sentiment d'extase, de sortie de soi ; car l'auteure n'est plus que contenant, n'ayant plus conscience d'elle-même en tant que sujet. Mais Johnson (1992, 21-22) insiste sur la capacité de la métaphore CONTENANT à exprimer l'expérience du mouvement dedans/dehors et l'interaction perceptuelle, en l'occurrence la mise en relation woolfienne entre perception de soi et perception des éléments extérieurs.
} 
schéma, selon lequel «nous (tous les êtres humains) -- sommes connectés avec ceci : que le monde entier est une oeuvre d'art, que nous faisons tous partie de cette œuvre d'art» :

From this I might reach a philosophy; at any rate it is a constant idea of mine; that behind the cotton wool is hidden a pattern, that we - I mean all human beings - are connected with this; that the whole world is a work of art; that we are parts of the work of art. (MB 1976, 72, je souligne)

Cet état de conscience, souvent provoqué dans Mrs Dalloway par la manifestation du temps, correspond à la définition donnée par le neurologue Damasio de la conscience étendue, extended consciousness, qu'il oppose à l'état de conscience-noyau (core consciousness) :

I have given names to those two kinds of consciousness. The minimal-scope kind I call core consciousness, the sense of the here and now, unencumbered by much past and by little or no future. It revolves around a core self and is about personhood but not necessarily identity. The bigscope kind I call extended or autobiographical consciousness, given that it manifests itself most powerfully when a substantial part of one's life comes into play and both the lived past and anticipated future dominate the proceedings. (...) Here the conscious mind widens and encompasses actual as well as imaginary contents effortlessly. (2012, 168-169)

Or, pour Damasio aussi, cet état supérieur de conscience s'accompagne d'un gain de sens, et le neurologue recourt au même terme, pattern, pour le définir :

the autobiographical self occurs when objects in one's biography generate pulses of core self that are, subsequently, momentarily linked in a largescale coherent pattern. (2012, 181, je souligne)

Deux aspects sont essentiels : la signification que prend soudain le sentiment de soi, mais avec cette nuance paradoxale que le soi s'y décentre, hors du présent, hors d'une perception linéaire du temps.

Le linguiste Johnson (1992, 19) utilise un terme synonyme de pattern, schema, qu'il définit comme une structuration issue de l'expérience et lui donnant sens : my use of the term focuses on embodied patterns of meaningfully organized experience. 
À la lecture de Mrs Dalloway et de quelques essais autobiographiques de Woolf, il est tentant ${ }^{9}$ de voir en la métaphore récurrente de l'eau pour exprimer cette intensité rare de conscience l'expression linguistique de ce que le psychologue Barsalou (1999) appelle un système symbolique perceptuel (Perceptual Symbol System) : une expérience sensorielle forte s'ancre si bien dans nos schémas neuronaux qu'en résulte un circuit la modélisant dans la structure profonde, stockée, de notre cerveau, constituant ainsi un système perceptuel prêt à être réactivé si une expérience est vécue sur le même mode. Il y a simulation suite à la formation et au stockage d'un schéma (le même mot pattern est utilisé) perceptuel dans la mémoire :

Because a perceptual symbol is an associative pattern of neurons, its subsequent activation has dynamical properties (Barsalou 1999, 584, je souligne).

La métaphore du plongeon et tout ce qui s'en rapproche, sentiment d'immersion, d'entrée dans l'eau fraîche, est l'expression linguistique la plus précise d'un quale essentiel au système cognitif de Woolf vécu comme un état d'hyperconscience. La romancière transmettrait ce quale ainsi que sa transposition stylistique à Clarissa, mais aussi à Septimus et Peter Walsh.

Barsalou (2012) montre également l'importance du langage dans la modélisation de ce système perceptuel : la perception sera renouvelée si les mots existent pour l'exprimer. Or cela, Woolf le constate : la réalité de la perception, la capacité à imprimer le choc/moment d'être existentiel ${ }^{10}$, à en faire un fondement signifiant de la mémoire, passe par la capacité à le mettre en mots (je poursuis la citation et souligne) :

it is a token of some real thing behind appearances and I make it real by putting it into words (MB 1976, 72)

9 Le même type de lien pourtant peu recommandable entre la femme et son oeuvre est encouragé par une parenthèse de la voix narrative à propos de Mrs Dalloway (her favourite reading as a girl was Huxley and Tyndall, and they were fond of these nautical metaphors, 85), dont le contenu fait encore écho aux métaphores employées par Woolf dans son Journal pour décrire notre rapport " quotidien " à la réalité : we are sealed vessels afloat on what is convenient to call reality (W 1976, 122, je souligne).

10 L'un des trois exemples que Woolf donne de ce choc est l'annonce du suicide de quelqu'un de son entourage, ce qui arrive à Clarissa à la fin du roman $(1976,72)$. 
Woolf fait elle-même le lien entre le caractère exceptionnel et atemporel de certains souvenirs et sa pratique de romancière consistant à ancrer la vie d'un personnage dans une scène originelle issue du passé, ce qu'elle appelle scene making :

But whatever the reason may be, I find that scene making is my natural way of marking the past. Always a scene has arranged itself: representative; enduring. This confirms me in my instinctive notion: (it will not bear arguing about; it is irrational) the sensation that we are sealed vessels afloat on what it is convenient to call reality; and, at some moments, the sealing matter cracks; in floods reality; that is, these scenes - for why do they survive undamaged year after year unless they are made of something comparatively permanent? Is this liability to scenes the origin of my writing impulse? (MB 1976, 122)

Pour Clarissa Dalloway, la scène originelle devenue système symbolique perceptuel sans cesse réactivé est ce contact avec les éléments exaltant le sentiment de soi, qu'elle expérimenta enfant à Burton :

Mrs. Dalloway said she would buy the flowers herself.

For Lucy had her work cut out for her. The doors would be taken off their hinges; Rumpelmayer's men were coming. And then, thought Clarissa Dalloway, what a morning - fresh as if issued to children on a beach.

What a lark! What a plunge! For so it had always seemed to her, when, with a little squeak of the hinges, which she could hear now, she had burst open the French windows and plunged at Bourton into the open air. (D 3)

La raison pour laquelle les autres protagonistes ont recours au même type de schéma perceptuel, plongeon et sentiment d'expansion de soi, reste hypothétique. Mais on a bien affaire à ce que le linguiste cognitiviste Grady (1997) caractérise comme une métaphore "primaire ", ou "primitive ", structurante : le moment de vie est éprouvé, dès l'enfance, comme un contact brutal et revigorant, vitalisant avec l'élément air ou eau.

\section{Confluence temporelle : a-temporalité de NOW}

Ainsi la métaphore du plongeon relie le présent de Clarissa à son passé de jeune fille ; le lecteur est à Londres et à Bourton, à la ville et à la plage : which she could hear now. Ouvrir la porte et plonger dans le frais matin londonien, c'est être de nouveau la jeune fille ouvrant les volets de la demeure familiale de Bourton et inspirant l'air iodé, c'est entendre de nouveau le fracas des vagues et ressentir le ravissement de faire partie d'un tout qui nous englobe et nous dissout. 
L'adverbe now fait ici paradoxalement office de connecteur entre situation présente et souvenir passé : c'est au présent de sa conscience élargie que Clarissa entend le bruit venu du passé ; les flux temporels se rejoignent dans l'émergence du schéma perceptuel. Now, employé 169 fois dans le roman, permet parfois à Woolf, comme le remarque De Mattia (2001, 237), de glisser du récit extérieur à l'intérieur d'une conscience ; il révèle la porosité des plans énonciatifs et l'instabilité temporelle ou logique de la voix narrative. Le repère, la situation d'énonciation dite prototypiquement par now s'élargit et se dissout dans une temporalité non linéaire. Now dépasse le maintenant, il transcende le hic et nunc. L'origine se perd dans la dilatation du soi ; et now exprime ainsi la confluence des flux temporels.

Now est également présent dans la citation récurrente que Woolf fait d'Othello (II,1) If it were now to die, 'twere now to be most happy, où le premier now est ancré dans l'expérience d'un bonheur vécu comme excessif (Othello retrouve sa douce épouse sur le sol chypriote après une traversée dangereuse de la Méditerranée), et le deuxième postule un moment hypothétique, post-mortem, d'éternité. Ici encore, now est le moins ancré dans le présent. La première citation d'Othello dans le texte intervient juste après un emploi similaire de now, dont la réitération fait resurgir (au présent) le sentiment passé éprouvé pour Sally Seton :

No, the words meant absolutely nothing to her now. She could not even get an echo of her old emotion. But she could remember going cold with excitement, and doing her hair in a kind of ecstasy (now the old feeling began to come back to her, as she took out her hairpins, laid them on the dressing-table, began to do her hair) with the rooks flaunting up and down in the pink evening light, and dressing, and going downstairs, and feeling as she crossed the hall 'if it were now to die 'twere now to be most happy'. (D 37-38)

Peut-être est-ce la répétition même de now, comme dans Othello, qui réalise ce décrochage de la situation d'énonciation ; il faudrait alors comprendre now now comme une sorte de corrélation performative ${ }^{11}$ signalant ou réalisant l'élargissement de la conscience.

11 Au sens large où De Mattia utilise la notion $(2001,255)$. 


\section{Métaphore : non linéarité dans le « courant " de conscience}

\section{Une opération cognitive parallèle...}

Comment l'écriture, nécessairement linéaire et construite, peut-elle rendre compte d'un état de conscience a-verbal et non linéaire, où stimuli internes, externes, présent et passé coexistent ? Le problème est exposé par Hogan $(2013,163)$ :

in a literary work, the parallelism of stream of consciousness must be serialised, as non-subvocalised experiences are presented in the sequence of speech.

Or, comme Hogan (2013, 154) l'explique et comme l'écriture de Woolf le fait percevoir, un processus cognitif typique implique des opérations parallèles :

parallelism is in fact the ordinary mode of cognitive operation (...) for the most part, it is not self-conscious. (...) most operations of the brain involve complex parallel operations across many neurons in different regions.

La métaphore, qui établit un parallèle entre deux domaines sémantiques, rend adéquatement ce processus : pour la métaphore du plongeon de l'incipit, le parallèle se dédouble : puisque le domaine cible est d'abord l'air du matin à Londres, puis, grâce au glissement temporel réalisé par now, l'air du matin à Bourton. L'opération métaphorique est ici doublement parallèle.

\section{....où la première personne éprouve un quale sans le dire explicitement}

La tâche de l'écrivain qui veut transcrire ce matériau spécifique qu'est la conscience est ardue. Joyce résout le problème par une technique de courant de conscience à la syntaxe chaotique, le moins construite possible. Woolf place au centre des consciences élargies de ses protagonistes des réseaux métaphoriques souterrains (pour prolonger sa propre métaphore de caverne) qui nous donnent un accès immédiat, par moments dilatés, à l'unicité d'une expérience sensorielle livrant une vision $\mathrm{du}$ monde. Cette vision/perception du monde est subjective mais n'emprunte pas pour autant les marques ordinaires de la subjectivité.

La métaphore fait cela, car aucune personne grammaticale n'en revendique l'origine : elle dissimule la prise en charge du discours et ne fait que livrer la vérité sensorielle d'une fusion cognitive. Roméo ne dit pas 
I love Juliet, mais Juliet is the sun. Woolf n'écrit pas "L'air frais du matin fit l'effet à Clarissa d'un plongeon dans l'eau fraîche ", mais What a plunge, où nul sujet, narrateur ou personnage, ne s'approprie le choc de l'expérience, auquel le lecteur est livré à son tour, dans une pure jouissance mimétique. C'est une réalisation littéraire possible de ce que Banfield (1982) appelle les "phrases indicibles " ou "phrases sans parole " (unspeakable sentences) : afin de représenter une conscience "non réflexive ", la voix narrative s'efface afin d'exprimer le point de vue non verbalisable de la première personne. La métaphore est ainsi largement responsable de ce que De Mattia $(2001,243)$ nomme un "effet de DIL" ou «DIL illocutoire ", c'est-à-dire la création d'une expressivité non pas du dire mais du faire, non pas du contenu de pensée mais du sentiment occupant le personnage alors sujet de conscience.

La métaphore What a plunge n'explicite aucun lien, fait l'ellipse du verbe et du sujet, ne désigne pas le domaine cible, car Clarissa est trop impliquée dans la situation pour exercer un esprit analytique. Le lecteur plonge avec elle. La syntaxe imite le rapport au réel ${ }^{12}$, et l'implication sensorielle de Clarissa se dit aussi dans la forme synthétique, elliptique de l'exclamative. Peut-être s'approche-t-on ici au plus près de ce que serait une donnée immédiate de la conscience (toujours fictive, bien sûr), peutêtre expérimente-t-on ici, pour notre plus grand plaisir, ce que devrait faire le stream of consciousness pour Woolf, siéger au centre de la conscience de l'esprit d'un autre ${ }^{13}$. Y plonger, corps et âme. Si Clarissa vit là où Septimus meurt, c'est peut-être parce qu'elle a compris la vertu de la sublimation esthétique, et plonge métaphoriquement, par l'âme, dans les choses, les êtres et la vie, là où le jeune homme plonge de tout son corps du haut de sa fenêtre : But this young man who had killed himself-had he plunged holding his treasure? (D 202)

12 Je poursuis encore en cela l'analyse de De Mattia (2001, 245).

13 Dans le Times Literary Supplement du 13 février 1919, où Woolf, dans une critique anonyme du roman de Dorothy Richardson, The Tunnel, explique ce que devrait faire, si elle était réussie, la méthode du stream of consciousness : make us feel ourselves seated in the centre of another's mind.

http://xroads.virginia.edu/ class/workshop97/gribbin/tunnel.html 


\section{« Nœud " de conscience : au plus subjectif, l'impersonnel}

\section{Fendre le temps du "je "}

Selon Damasio (2012, 157), la conscience est irréductiblement subjective, et ne peut être éprouvée que dans la "perspective exclusive de la première personne"; pourtant cette perspective se saisit dans une expansion de la conscience dans le temps, dans une ouverture subite que la métaphore woolfienne de la "fente" (split) exprime bien, à propos de Septimus: The word 'time' split its husk, poured its riches over him (D 76 , je souligne). Et lors de l'épiphanie homosexuelle de Clarissa déjà citée, où la même métaphore apparaît : some pressure of rapture, which split its thin skin and gushed and poured (D 34-35, je souligne).

Dans les deux cas, la métaphore split est suivie d'une autre, pour, se déverser, les deux exprimant un bonheur des sens et du sens transcendantal qui dépasse le soi, le je ; le référent du possessif its est d'ailleurs peu clair, la peau est sans doute métaphorique, sans doute s'agitil de l'enveloppe, l'écorce du monde, ou bien du ravissement; le flottement référentiel, l'indistinction sont essentiels, car celle qui ressent n'est plus que déhiscence sensorielle, accueil des autres éléments confondus. Lorsque Peter fait l'expérience heureuse du moment signifiant, de plus en plus fréquent à mesure qu'il vieillit, il ressent ce plaisir et ce sens comme de moins en moins personnels :

every ounce of pleasure, every shade of meaning; which both were so much more solid than they used to be, so much less personal (D 87)

La conscience chez Woolf s'apprécie dans la dissolution du soi. Cela semble cohérent si l'on considère ses préférences stylistiques : déclarations d'aversion pour le pronom "je " et goût pour l'emploi récurrent du pronom one ${ }^{14}$ :

One began to be tired of ' $I$ '. (...) why was I bored? Partly because of the dominance of the letter ' $\mathrm{I}$ ' and the aridity, which, like the giant beech tree, it casts within its shade. Nothing will grow there. (R 1984, 93-94)

Le "je " est aride, car il ne permet pas l'éclosion du sens. Woolf raconte avoir connu l'un de ses premiers chocs lorsqu'elle fit la

${ }_{14}$ Voir Mignot (2016) et De Mattia (2001, 260). 
découverte, enfant, regardant un parterre de fleurs, de l'appartenance de la fleur à la terre, la fleur était en partie fleur, et en partie terre (MB 1976, 71) : that was the real flower; part earth; part flower. Elle se promit de mettre ce savoir précieux de côté. Lorsque l'image de l'eau, de l'immersion est convoquée dans Mrs Dalloway, c'est un soulagement, une délivrance : le corps fait enfin partie d'un plus grand corps qui l'englobe ${ }^{15}$, et lui donne sens.

\section{Le " noud " de conscience et le soi " diamant "}

Plutôt que la métaphore du "flux" de conscience, Woolf emploie dans son essai The Moment: Summer's Night (1938) celle du " nœud" de conscience, où communient quatre personnes dont les quatre corps sont noués, sombrant avec les chaises sur lesquelles ils sont assis, vers le centre de la terre. Ici encore, le moment d'étant est co-occurrent d'une métaphore d'entrée dans l'eau et aussi d'appartenance au monde (naturel ou social) :

this moment is also composed of a sense that the legs of the chair are sinking through the centre of the earth, passing through the rich garden earth; they sink, weighted down. (...) One becomes aware that we are spectators and also passive participants in a pageant. (...) But that is the wider circumference of the moment. Here in the centre is a knot of consciousness; a nucleus divided up into four heads, eight legs, eight arms, and four separate bodies. (CE 1966, 293-94)

$\mathrm{Au}$ centre de la conscience, il y a diffraction, division du soi ; le noyau se sépare, entrelaçant plusieurs perceptions. La métaphore du "nœud" de conscience collectif, sombrant vers le centre de la terre, est une autre expression de ce désir de "connexion" entre les "cavernes" exprimé par Woolf dans son Journal (déjà cité 1959, 60-61).

Clarissa médite à plusieurs reprises sur l'éparpillement de soi. Qui est-elle? Est-elle la femme qui reçoit ce soir, Clarissa, elle-même ? Les verbes collect et assemble sont utilisés pour décrire l'effort réflexif de saisie

15 Pensant à sa mort, Clarissa se console en s'imaginant continuer à exister quelque part, dans les rues de Londres, dans le flux et reflux des choses (métaphore aquatique) : somehow in the streets of London, on the ebb and flow of things, here, there, she survived, Peter survived, lived in each oter she being part, she was positive, of the trees at home; of the house there, ugly, rambling all to bits and pieces as it was; part of people she had never met (D 9-10, je souligne). C'est le prédicat <be part of $>$, utilisé quatre fois dans le roman, qui exprime le mieux cette appartenance bienfaisante. 
objective de soi, qu'elle active spontanément lorsqu'elle se regarde ou se surprend dans le miroir, unifiée et pointue :

collecting the whole of her at one point (as she looked into the glass) (...) She pursed her lips when she looked in the glass. It was to give her face point. That was her self-pointed; dartlike; definite. That was her self when some effort, some call on her to be her self, drew the parts together, she alone knew how different, how incompatible and composed so for the world only into one centre, one diamond, one woman who sat in her drawing-room and made a meeting-point, a radiancy no doubt in some dull lives, a refuge for the lonely to come to, perhaps; (...). Strange, she thought, pausing on the landing, and assembling that diamond shape, that single person, strange how a mistress knows the very moment, the very temper of her house! (D 40-41, je souligne)

Seule Clarissa sait à quel point ce n'est pas elle, ce centre solide et univoque, qu'elle appelle "diamant», et qui semble cette fois-ci correspondre au soi noyau défini par Damasio, ancré dans l'ici et maintenant ; cette image "objective" (objectale) d'elle-même est au plus loin de l'état diffus de conscience où la réalité des êtres se révèle ; c'est le rôle social qu'elle excelle à jouer, le soi-objet dont on peut délimiter les contours abrupts et taillés dans la pierre.

Le noyau dur, identité sociale de Clarissa Dalloway en parfaite mondaine, résulte donc d'un processus d'assemblage contraint et réflexif, qui réunit les différentes parties de soi. Il est aux antipodes du ravissement, sortie de soi éprouvée par le personnage lors des extases momentanées que lui procure la dissolution dans le cours des choses, trafic tumultueux de la ville, expérience a-temporelle d'un moment pourtant situé.

\section{Autre marqueur de conscience élargie :-ING participial}

L'étude de ces moments d'intensité dans Mrs Dalloway, dilatation $\mathrm{du}$ temps et de soi, révèle donc quelques marqueurs récurrents, dont l'adverbe now et les métaphores autour de l'eau ; un autre marqueur de conscience élargie est le morphème lié -ING, dont on note également la présence dans le SN moment of being.

L'emploi excessivement fréquent de -ING, d'après nos calculs, est un trait spécifique de Mrs Dalloway par rapport au corpus de comparaison. Le score de spécificité donné par TXM est 
remarquablement élevé : 15,1 . Or beaucoup de ces occurrences semblent ${ }^{16}$ être des emplois participiaux, où -ING est tête d'une proposition participiale détachée dont le sujet n'est pas exprimé explicitement, comme dans ce passage suivant immédiatement la métaphore du plongeon à l'incipit du roman :

How fresh, how calm, stiller than this of course, the air was in the early morning; like the flap of a wave; the kiss of a wave; chill and sharp and yet (for a girl of eighteen as she then was) solemn, feeling as she did, standing there at the open window, that something awful was about to happen; looking at the flowers, at the trees with the smoke winding off them and the rooks rising, falling; standing and looking until Peter Walsh said, 'Musing among the vegetables?'-was that it? - (D 3)

Feeling, standing et looking sont tous trois têtes de participiales dont le sujet she est certes récupérable, mais disséminé, éparpillé dans le contexte, et la distance à l'identité du référent est encore signifiée par le marqueur d'indéfini a dans le $\mathrm{SN}$ a girl of eighteen; le support que les adjectifs chill and sharp and yet solemn qualifient est de moins en moins net, jusqu'à l'hypallage où le sentiment de Clarissa jeune fille est indistinguable de la sensation de l'air frais ; puis le sujet grammatical she disparaît dans la perception des arbres, des corbeaux ; il n'est pas rappelé lors de la reprise de standing et looking après les relatives elliptiques winding off them et rising, falling, où l'ellipse de la relation d'identification est une autre manifestation de l'expérience sensorielle synthétique du sujet de conscience. -ING participial est un élément stylistique essentiel dans l'écriture de Woolf, une autre réalisation linguistique de la dissolution du soi dans ces moments de conscience élargie ${ }^{17}$. Forme non-finie, la base verbale portant -ING permet d'échapper à un ancrage temporel spécifique et contribue à désigner un moment de suspension, hors du temps chronologique ou énonciatif.

16 Je n'ai pu à ce jour en faire la preuve statistique rigoureuse, mes capacités d'exploitation du logiciel TXM n'étant pas encore suffisantes, et la discrimination, par l'outil informatique, du -ING participial tête de proposition adverbiale, peut-être impossible à distinguer des autres formes verbales en -ING, notamment des participes présents adjectivaux.

17 Le même phénomène (avalanche de participiales en -ING dont le sujet est de plus en plus difficile à récupérer) est visible dans notre extrait cité p. 6 de cette étude (38 dans notre édition de Mrs Dalloway) où le connecteur and, de manière atypique, n'entraîne pas la mention du sujet grammatical du verbe tête de la subordonnée : and dressing, and going downstairs, and feeling (...). 


\section{Conclusion : le sens du moment (mouvement et émotion)}

Dans Mrs Dalloway, le temps n'existe que sous forme de moment(s). Moment est utilisé au singulier dans la plupart des occurrences $(79 \%)$. La conscience, ni le temps, ne s'écoulent en flots continus; mais leur rencontre provoque des prises de conscience menant à la sortie de soi, qui est sens et sentiment de plénitude existentielle. Le moment est ici mouvement, comme dans l'étymon latin momentum, moment décisif, formé sur le verbe movere ; un moment, le temps se fait mouvement, perpétué dans les métaphores dynamiques d'entrée dans l'eau, de vague qui se répand. Si le temps ne peut être conçu par l'esprit, le corps l'entend qui sonne et l'accueille inconsciemment, et les flots de son temporel provoquent un décentrement salutaire, identitaire et esthétique. Peut-être ne peut-on ressentir autrement le temps qui passe ; peut-être l'émotion (de movere également) excessive alors éprouvée, extase et ravissement dépassant le sujet de conscience vers la conscience de soi dans le monde, est-elle mouvement de l'âme acceptant l'abandon au temps. 


\section{BIBLIOGRAPHIE}

\section{SOURCES PRIMAIRES}

DICK, Susan. (ed.). 1989. The Complete Shorter Fiction of Virginia Woolf. Londres: The Hogarth Press. (abrégé ci-dessus en CSF)

SCHULKIND, Jeanne. (ed.). 1976. Virginia Woolf: Moments of Being Unpublished Autobiographical Writing. New York: Harcourt Brace Jovanovich. (abr. MB)

WOOLF, Virginia. 1959. A Writer's Diary. Londres: The Hogarth Press. (abr. W)

WOOLF, Virginia. 1966. Collected Essays, Vol. 2. Londres: The Hogarth Press. (abr. CE)

WOOLF, Virginia. 1984 [1929]. A Room of One's Own and Three Guineas. Londres: The Hogarth Press. (abr. R)

WOOLF, Virginia. 2000 [1925]. Mrs Dalloway. Londres: Penguin Classics. (abr. D)

\section{SOURCES SECONDAIRES}

BANFIELD, Ann. 1982. Unspeakable Sentences: Narration and Representation in the Language of Fiction. Boston: Routledge \& Kegan Paul.

BARSALOU, Lawrence W. 1999. "Perceptual symbols systems ". Behavioral and Brain Sciences 22: 577-660. 
BARSALOU, Lawrence W. \& GENDRON, Maria, LINDQUIST, Kristen A., FELDMAN BARRETT, Lisa. 2012. "Emotion words shape emotion percepts ". American Psychological Association 12.2: 314-325.

BERGSON, Henri. 1922. Durée et simultanéité : à propos de la théorie d'Einstein. Paris : Alcan.

BOWLING, Lawrence E. 1950. "What is the stream of consciousness technique? ». PMLA 65.4: 333-345.

DAMASIO, Antonio. 2012. Self Comes to Mind: Constructing the Conscious Brain. Londres: Vintage.

DE MATTIA, Monique. 2001. "Mrs Dalloway de Virginia Woolf ou l'instabilité du discours rapporté ". In M. De MATTIA et A. JOLY (eds), De la syntaxe à la narratologie énonciative. Paris : Ophrys, p. 227-264.

DE MATTIA, Monique. 2006. Le discours indirect libre au risque de la grammaire : le cas de l'anglais. Aix-en-Provence : Presses Universitaires de Provence.

FAUCONNIER, Gilles. 1994. Mental Spaces: Aspects of Meaning Construction in Natural Language. New York: Cambridge University Press.

GOODMAN, Nelson. 1975. "The status of style». In Critical Inquiry $1.4: 799-811$.

GRADY, Joseph. 1997. Foundations of Meaning: Primary Metaphors and Primary Stress. PhD. http://escholarship.org/uc/item/3g9427m2, consulté le 8 juillet 2016.

GUTTENPLAN, Samuel. 2005. Objects of Metaphor. Oxford: Clarendon Press.

HOGAN, Patrick C. 2003. Cognitive Science, Literature and the Arts. New York: Routledge. 
HOGAN, Patrick C. 2013. "Parallel processing and the human mind: Reunderstanding consciousness with James Joyce's Ulysses ». Journal of Literary Semantics 42.2: 149-164.

JAMES, William. 1998, 1890. The Principles of Psychology. Bristol: Thoemmes; Tokyo: Maruzen.

JAMES, William. 1912. Essays in Radical Empiricism. Londres: Longmans, Green and Co.

JAMES, William. 1916, 1983. Talks to Teachers in Psychology; and to Students on Some Life's Ideals. Cambridge, London: Harvard University Press.

JOHNSON, Mark. 1992. The Body in the Mind: The Bodily Basis of Meaning, Imagination, and Reason. Chicago, London: Chicago University Press.

LACEY, Simon, STILLA, Randall \& SATHIAN, Krishnankutty. 2012. "Metaphorically feeling: Comprehending textural metaphors activates somatosensory cortex ». Brain\&Language 120: 416-421.

LANGACKER, Ronald W. 2009. Investigations in Cognitive Grammar. Moutin de Gruyters: Berlin.

LAKOFF, George \& JOHNSON, Mark. 1980. Metaphors We Live By. Chicago, Londres: University of Chicago Press.

LAKOFF, George. 1993. "The contemporary theory of metaphor ». In A. ORTONY (ed.) Metaphor and Thought. Cambridge: Cambridge University Press.

LOWE, Ronald. 2007. Introduction à la psychomécanique du langage. Vol. I. Québec : Presses de l'Université Laval.

MIGNOT, Elise. 2016. "Le pronom personnel one dans $A$ Room of One's Own de Virginia Woolf. Engagement ou désengagement?", Etudes de Stylistique Anglaise 9, "Engagement/Commitment": 9-31. 
NEVEUX, Julie. 2013. Le sentiment dans la langue. Préface de P. COTTE. Paris : Éd. Rue d'Ulm.

NEVEUX, Julie. 2014. "Métaphore grammaticale : le nom en -ness, une création lexicale à usage unique". Lexis 8 , http://lexis.univlyon3.fr/spip.php?article204, consulté le 8 juillet 2016.

POLVINEN, Meria. 2013. "Affect and artifice in cognitive literary theory ". Journal of Literary Semantics 42.2: 165-180

PUGMIRE, David. 1998. Rediscovering Emotion. Edimbourg: Edinburgh University Press.

RICOEUR, Paul. 1984. Temps et récit 2; La configuration dans le récit de fiction. Paris : Éd. du Seuil. "Entre le temps mortel et le temps monumental : Mrs Dalloway", p. 152-167.

SWEETSER, Eve \& DANCYGIER, Barbara (eds). 2012. Viewpoint in Language; a Multimodal Perspective. Cambridge, New-York: Cambridge University Press.

VERDONK, Peter. 2013. The Stylistics of Poetry: Context, Cognition, Discourse, History. Londres: Bloomsbury. 